\title{
Frequency-Domain Deconvolution for Flight Dynamics Applications
}

\author{
Jared A. Grauer* \\ NASA Langley Research Center, Hampton, Virginia, 23681 \\ and \\ Matthew J. Boucher ${ }^{\dagger}$ \\ NASA Armstrong Flight Research Center, Edwards, California, 93523
}

\begin{abstract}
A frequency-domain deconvolution method is presented for estimating input data from measured output data and a model of the dynamic process. The method combines Optimal Fourier Smoothing, a Wiener filter, and a high-accuracy Fourier transform. Linear timeinvariant systems are considered, which may be stable or unstable, open-loop or closed-loop, and single-input single-output or multiple-input multiple-output. Simulation data were used to demonstrate the method and investigate robustness to measurement noise and modeling error. Flight test examples include integrating noisy data, synchronizing time series with relative time skews, reconstructing control surface deflections, and extracting velocity components of turbulence. Results indicated that the method performs well when the model is sufficiently accurate and when the signal can be distinguished from noise.
\end{abstract}

\section{Nomenclature}

\begin{tabular}{llll}
$a_{z}$ & vertical accelerometer output, $\mathrm{g}$ & $\omega$ & \multicolumn{1}{l}{ frequency, rad/s } \\
$b$ & wingspan, $\mathrm{ft}$ & $\omega_{N}$ & Nyquist frequency, rad/s \\
$\bar{c}$ & mean aerodynamic chord, $\mathrm{ft}$ & $\omega_{n}$ & natural frequency, rad/s \\
$j$ & imaginary number, $=\sqrt{-1}$ & \multicolumn{2}{c}{ nubscripts } \\
$p, q, r$ & body-axis angular rates, rad/s & 0 & reference value \\
$R^{2}$ & coefficient of determination & $c m$ & center of mass location, in \\
$S$ & wing reference area, $\mathrm{ft}^{2}$ & & \\
$s$ & Laplace variable & Superscripts \\
$t$ & time, s & -1 & inverse \\
$u, v, w$ & body-axis velocity components, $\mathrm{ft} / \mathrm{s}$ & $\cdot$ & time derivative \\
$\alpha$ & angle of attack, rad & $\dagger$ & complex conjugate transpose \\
$\alpha_{g}$ & angle of attack gust, rad & - & estimate \\
$\Delta$ & perturbation or determinant & $|\cdot|$ & magnitude or absolute value \\
$\delta_{e}$ & elevator deflection, rad & & \\
$\zeta$ & damping ratio & &
\end{tabular}

\footnotetext{
* Research Engineer, Dynamic Systems and Control Branch, MS 308, Senior Member AIAA.

$\dagger$ Research Engineer, Controls and Dynamics Branch, MS 4840D, Member AIAA.
} 


\section{Introduction}

$\mathrm{T}^{\mathrm{N}}$ some situations, an analyst may find that measured data are corrupted by the unwanted filtering effects of 1 an additional dynamic system. This filtering can potentially make the observed measurement significantly different than the signal of interest. For example, a seismogram will register seismic waves released during an earthquake, but the recorded time series data are attenuated and shifted according to the relative location of the instrument to the epicenter, and other factors.

A similar situation may arise in the flight testing of aerospace vehicles, where the dynamic systems involved can represent the flight dynamics of the vehicle, or the sensor dynamics of transducers used to observe the vehicle motion, or other phenomena. Considering sensor dynamics, most flight test experiments are designed such that dynamic responses of the instrumentation are negligible over the bandwidth of interest and can be safely ignored during analysis. If adequate sensors were not used, the measured signals can be significantly different than those sought, which can degrade the accuracy of ensuing analyses. If the experiment cannot be redesigned and repeated, perhaps due to budget constraints or loss of aircraft, deconvolution techniques can sometimes be applied to reduce the unwanted effects of the sensor dynamics on the measured data and improve analysis results.

Deconvolution can be explained in terms of its inverse operation, convolution. When a linear dynamic system is excited by an input, the output is defined by a convolution of the input with the system impulse response function. Deconvolution is the reverse operation, in which the influence of the system impulse response is removed from an output to yield the input. Besides removing unwanted filtering effects from measured data, deconvolution has several other applications such as sharpening digital images, removing distortions from optical data, and differentiating noisy data.

A method for the deconvolution of flight test data in the frequency domain is described in this paper. The method is based on Optimal Fourier Smoothing, developed in Ref. [1]. First, a Wiener filter [2] is designed to separate the measurement into signal and noise components. Techniques from Fourier analysis [3] were adopted to improve the accuracy of this step. The estimated signal is then passed through the inverted dynamics model and the input is reconstructed in the frequency and time domains. It was found using both simulation and flight test examples that the method performs well given an adequate model of the dynamic system and signal-to-noise ratios.

This paper is organized as follows. Section II explains the mathematical framework of the deconvolution problem. Section III describes the deconvolution method and its implementation. Section IV provides five examples. First, a simulation model is used to demonstrate the accuracy of the method and investigate robustness to measurement noise and modeling error. Flight test data from the X-56A and T-2 aircraft are used to show integration of noisy data, synchronization of time series having relative time skews, reconstruction of unknown control surface deflections, and extraction of turbulence velocity inputs. Lastly, conclusions are discussed in Section V.

\section{Problem Statement}

The measurement process is depicted in Fig. 1 as a block diagram. The signal of interest $u(t)$ is the input to a linear time-invariant system having frequency response $H(j \omega)$. The output of the system $y(t)$ can be solved for in the time domain using the convolution integral

$$
y(t)=\int_{0}^{t} u(\tau) h(t-\tau) d \tau
$$

where $h(t)$ is the impulse response function of the system, or in the frequency domain as

$$
Y(j \omega)=H(j \omega) U(j \omega)
$$

Lowercase and uppercase letters are used to denote Fourier transform pairs, e.g., $u(t)$ and $U(j \omega)$. A Gaussian noise process $n(t)$ is added to the output to model the recorded measurement

$$
z(t)=y(t)+n(t)
$$

For clarity, the method is presented using continuous signals and systems, although discrete versions may also be used. 


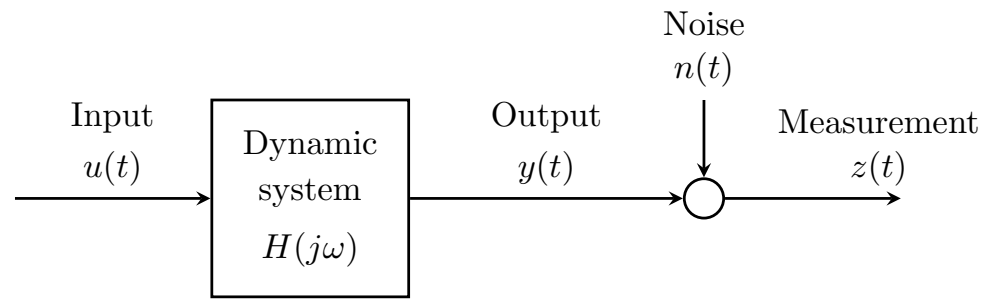

Figure 1. Measurement process block diagram

The goal of deconvolution is to reconstruct $u(t)$ from $z(t)$. The simplest deconvolution technique is called inverting the dynamics or inverse filtering. The measured data are transformed into the frequency domain, divided by a frequency response model of the dynamic system, and inverse-transformed back into the time domain. This method is generally unstable with respect to the input when the measured output data contain noise. For example, most physical systems act as low-pass filters, so that inverting their frequency response will amplify higher-frequency content having lower signal-to-noise ratios. Therefore, a Wiener filter is often used to remove the noise before inverting filter dynamics in a process called Wiener Deconvolution [2]. This idea has found successful applications in seismology, digital image processing, acoustics, and others. Blind deconvolution techniques can be applied if explicit knowledge of the dynamic system cannot be obtained [4]. In the related field of inverse simulation, optimization techniques are used to determine inputs from measured outputs, for example to determine control inputs of a helicopter to follow a specific trajectory [5].

\section{Method}

The method developed here is based on Wiener Deconvolution and a modification of Optimal Fourier Smoothing [1] for frequency-domain deconvolution applications. A block diagram of the process is depicted in Fig. 2. The upper, forward path shows the measurement process, repeated from Fig. 1. The lower, reverse path shows the reconstruction process, which is described in this section. A Wiener filter is first used to separate the measurement into output and noise components. The output estimate is then passed through the inverted model of the system dynamics to yield the input. The carets indicate that a variable is an estimate or model.

Because frequency-domain techniques are used, only linear time-invariant systems are considered. However, analysis in the frequency domain permits that the systems may be stable or unstable. Because only inputs and outputs are considered, the systems may describe open-loop or closed-loop dynamics. The deconvolution method is presented for single-input single-output systems, but extends also to multiple-input multiple-output systems, as explored later with the flight-test examples.

Computer software used for the Fourier transform and inverse transform, input design, signal processing, and system identification are available in a MATLAB ${ }^{\circledR}$ toolbox called System IDentification Programs for AirCraft, or SIDPAC, ${ }^{6}$ which is associated with Ref. [7].

\section{III.A. Wiener Filter Design}

The Wiener filter is used to separate the measured data into output and noise. The filter minimizes

$$
E(j \omega)=\int_{0}^{\omega_{N}}|Y(j \omega)-\hat{Y}(j \omega)|^{2} d \omega
$$

which is the mean squared error between the output and its estimate. By writing $E(j \omega)$ in terms of $Y(j \omega)$ and $N(j \omega)$, then setting the derivative equal to zero, the optimal filter equation is $[2,8]$

$$
\Phi(j \omega)=\frac{Y^{2}(j \omega)}{Y^{2}(j \omega)+N^{2}(j \omega)}
$$

This filter equation is the ratio of the output power spectrum to the the total power of the measured data.

The output and noise power spectra are required to form the Wiener filter but are not known (otherwise the filter would not be needed). However, relatively crude models of the output and noise can be inferred 


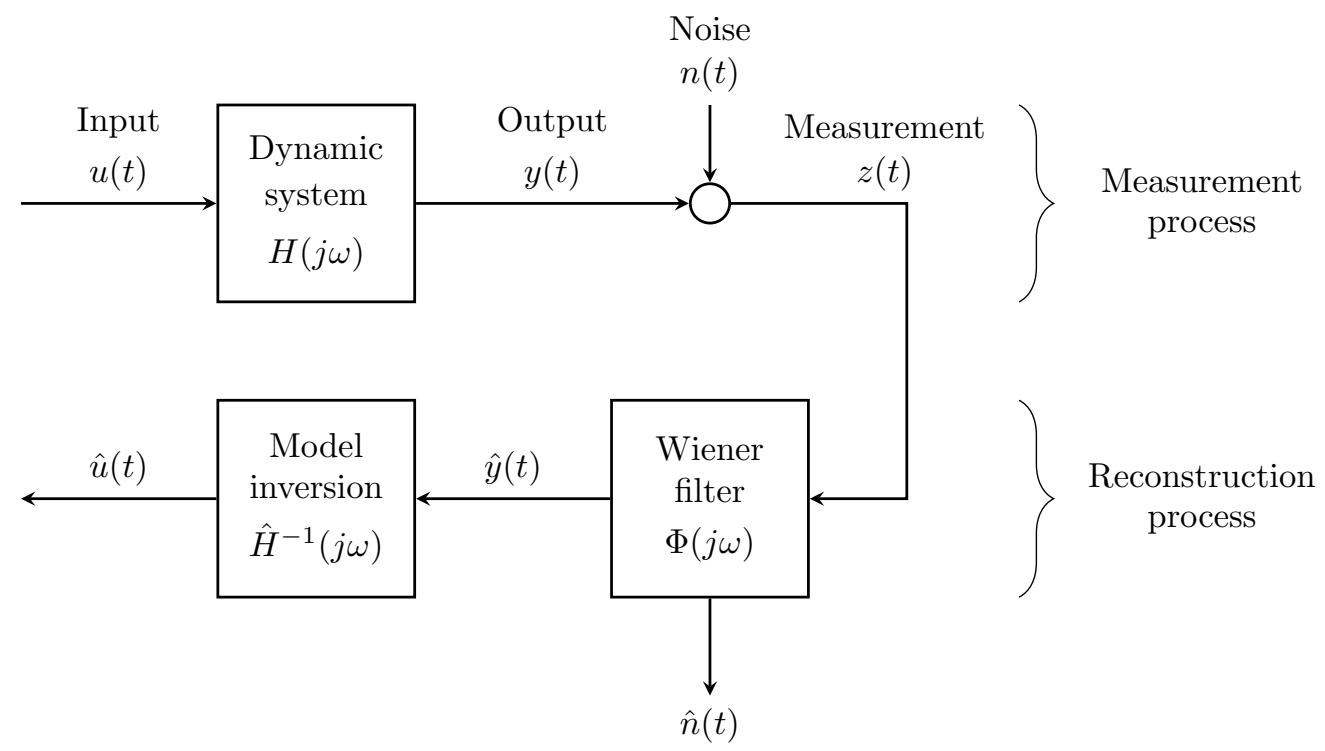

Figure 2. Wiener deconvolution block diagram

from the measurements and give good performance of the filter. Spectral data and models are depicted in Fig. 3. First, the magnitude spectrum for the measurements $|Z|$ is computed, as discussed in the next section. Then a noise model $|\hat{N}|$ is fit using data at higher frequencies, past the expected bandwidth of the output signal, and extrapolated to the lower frequencies. The difference between the computed measurement spectra and the extrapolated noise spectrum is the estimated output spectrum $|\hat{Y}|$. These simple models identified are used to define the Wiener filter $\Phi$, which varies with frequency between 0 and 1 .

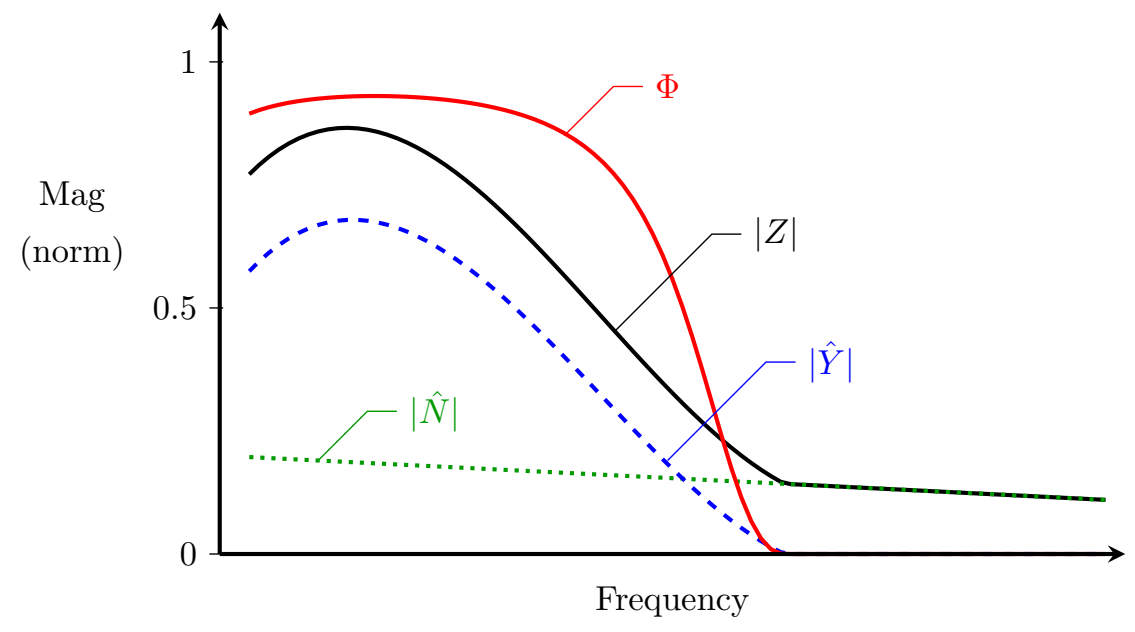

Figure 3. Wiener filter design from measurement spectrum

\section{III.B. Spectral Estimation}

A variety of methods [8-10] can be used to compute the measurement spectrum for designing the Wiener filter. Another approach, based on Fourier analysis, was introduced in Ref. [3] and was combined with the Wiener filter to solve smoothing and noise estimation problems for flight data analysis in Ref. [1]. A brief summary of the approach developed in Ref. [1] is presented here, with some further elaborations. The advantages of using this method, as will be discussed, are that an accurate estimate of the power spectrum is obtained without relying on analyst judgment or tuning parameters in the analysis, and that the contrast 
between the output signal power and the noise power is increased to more precisely model the power spectra. Accurate and distinct models for the signal and noise spectra lead to a highly effective Wiener filter, which gives the smooth and accurate output estimates, which are in turn required for good deconvolution results.

The derivation of the Fourier technique begins by noting that any analytic function can be expanded as the truncated Fourier series approximation

$$
z(t) \simeq \frac{a_{0}}{2}+\sum_{k=1}^{M} a_{k} \cos \left(\frac{\pi k}{T} t\right)+b_{k} \sin \left(\frac{\pi k}{T} t\right)
$$

over the domain $t \in[0, T]$, where $k$ is the harmonic number and $M$ is the number of harmonics included. As $M$ increases, the approximation of $z(t)$ becomes more accurate. If the measured time series $z(t)$ is reflected about the origin as an odd function $g(t)$ such that

$$
g(t)= \begin{cases}-z(-t), & \text { for } t \in[-T, 0) \\ z(t), & \text { for } t \in[0, T]\end{cases}
$$

then the cosine terms in the Fourier series representation of $g(t)$ evaluate to zero, because cosines are even functions whereas $g(t)$ is odd. The Fourier series simplifies to a sine series having the coefficients

$$
b_{k}=\frac{2}{\pi} \int_{0}^{T} g(t) \cdot \sin \left(\frac{\pi k}{T} t\right) d t
$$

where for convenience, the limits of integration have been reduced from $[-T, T]$ to $[0, T]$ because the function has odd symmetry. Applying integration by parts three times and evaluating boundary conditions, the sine series coefficients expand as [3]

$$
b_{k}=-\frac{2}{\pi}\left(\frac{T}{\pi k}\right)\left[(-1)^{k} g(T)-g(0)\right]+\frac{2}{\pi}\left(\frac{T}{\pi k}\right)^{3}\left[(-1)^{k} \ddot{g}(T)-\ddot{g}(0)\right]+\text { h.o.t. }
$$

where h.o.t. are neglected higher-order terms. The first term in Eq. (9), which is proportional to $k^{-1}$, dictates the rate of convergence for the series. For example, if the power spectrum of $g(t)$ abruptly goes to zero after a given frequency, as in an ideal low-pass filter, the Fourier sine coefficients decay in proportion to $k^{-1}$ thereafter.

If a trend line $\alpha+\beta t$ is removed from the data such that the endpoints $g(0)=g(T)=0$, then the first term in Eq. (9) evaluates to zero and the convergence rate of the series becomes $k^{-3}$. This faster rate of convergence provides a more accurate view of the spectrum and is useful for modeling the signal and noise spectra. This enhanced convergence results from removing the endpoint discontinuities, thereby creating a continuous and periodic signal more suited to the sinusoid basis functions of the Fourier series. When there are discontinuities in the values or derivatives at the endpoints, the convergence of the Fourier series is slower and the Wiener filter is less accurate. In conventional application of Fourier transforms (e.g., using the fast Fourier transform), a mean line or trend line is removed from the data, but not necessarily one that sets the endpoints to zero. This creates a discontinuity in the data and results in a convergence rate of $k^{-1}$ for its Fourier series representation.

Alternatively, $g(t)$ could have been constructed by reflecting $z(t)$ as an even function, instead of an odd one. This would result in a Fourier cosine series, but only a convergence rate of $k^{-2}$ due to a slope discontinuity at the endpoints [3]. One may also seek to further increase the convergence rate by removing discontinuities in the curvature of the series. However, this requires significant manipulation of the measured data using higher-order polynomial trend lines. In addition, this would smooth the second derivative, which is analogous to altering the forces acting on the dynamic system [3]. Convergence speeds of $k^{-3}$ represent a substantial improvement over the nominal $k^{-1}$, and require only small manipulations of the data which are routinely performed in frequency-domain analyses.

The point of applying the Fourier sine series in this way was to achieve a clear view of the power spectra for the measured data. The $k^{-3}$ convergence rate increases the contrast between signal and noise, so that accurate spectral models may be selected automatically [6] or more easily from visual inspection. 


\section{III.C. Implementation}

The Wiener filter was implemented in the frequency domain by multiplying the Fourier sine coefficients by the filter weighting as a function of frequency. The time-domain output was then constructed by synthesizing the Fourier sine series and restoring the trend line removed from the data, as

$$
\hat{y}(t)=(\alpha+\beta t)+\sum_{k=1}^{M} \Phi\left(j \omega_{k}\right) b_{k} \sin \left(\frac{\pi k}{T} t\right)
$$

The noise estimate was computed from the time-domain data by rearranging Eq. (3) as

$$
\hat{n}(t)=z(t)-\hat{y}(t)
$$

and substituting the measured data and reconstructed output. The model inversion is performed in the frequency domain as

$$
\hat{U}(j \omega)=\hat{H}^{-1}(j \omega) \hat{Y}(j \omega)
$$

where a high-accuracy Fourier transform $[6,11]$, based on the chirp-z transform, was used to transform the data into the frequency domain up to the Nyquist rate. The time-domain estimate of the input is then synthesized using the Fourier inverse transform

$$
\hat{u}(t)=\int_{0}^{\omega_{N}} \hat{U}(j \omega) e^{j \omega t} d \omega
$$

which in many applications can be implemented using a simple Euler approximation to the integral [11].

\section{Examples}

\section{IV.A. Simulation Results}

A simulation example was first used to demonstrate the method and assess robustness to measurement noise and modeling errors. The input to be reconstructed was $12 \mathrm{~s}$ in duration and was sampled at $50 \mathrm{~Hz}$. It included a $10 \mathrm{~s}$ random noise sequence with equal power between $0.1-5 \mathrm{~Hz}$, constructed using the Lánczos method [12].

The dynamic system was a second-order transfer function with a time delay

$$
H(s)=\frac{\omega_{n}^{2}}{s^{2}+2 \zeta \omega_{n} s+\omega_{n}^{2}} e^{-\tau s}
$$

which is a common description of a dynamic system. Nominal values of the parameters were selected as $\omega_{n}=18.8 \mathrm{rad} / \mathrm{s}(3 \mathrm{~Hz}), \zeta=0.5$, and $\tau=0.1 \mathrm{~s}$. In this nominal case, the bandwidth of the input extends beyond the bandwidth of the system, where lower frequencies are passed, those around $3 \mathrm{~Hz}$ are amplified, and those between $3-5 \mathrm{~Hz}$ are attenuated.

The output was summed with a second random noise sequence, which had equal power up to the $25 \mathrm{~Hz}$ Nyquist frequency. This measurement noise had a nominal standard deviation corresponding to $20 \%$ of the root mean squared variation in $u(t)$, which produced a signal-to-noise ratio of 25 for $z(t)$. Time histories of the input, output, noise, and measurement are shown in Fig. 4. The true values are shown in blue whereas the reconstructions (discussed next) are in red. The scale on the noise plot is an order of magnitude smaller than for the other plots to more easily distinguish between the true and reconstructed time series. Comparing $u(t), y(t)$, and $z(t)$, it is evident that the filtering effects of the dynamic system (e.g., amplitude attenuation, phase distortion, time delay) and the additive noise made the measurement significantly different than the input.

Fourier sine coefficients for the measured data are shown in Fig. 5 as gray impulses. The noise model (shown in green) was found by fitting a constant to the Fourier coefficients between 10-25 Hz. The output model (shown in blue) was a constant fit to the coefficients between $0-5 \mathrm{~Hz}$, and then linearly decreasing from that value to zero to approximate the $k^{-3}$ decrement. The resulting Wiener filter design (shown in red with axes on the right side) passes the signal below $5 \mathrm{~Hz}$ with only a small attenuation, removes the signal 
- Reconstruction
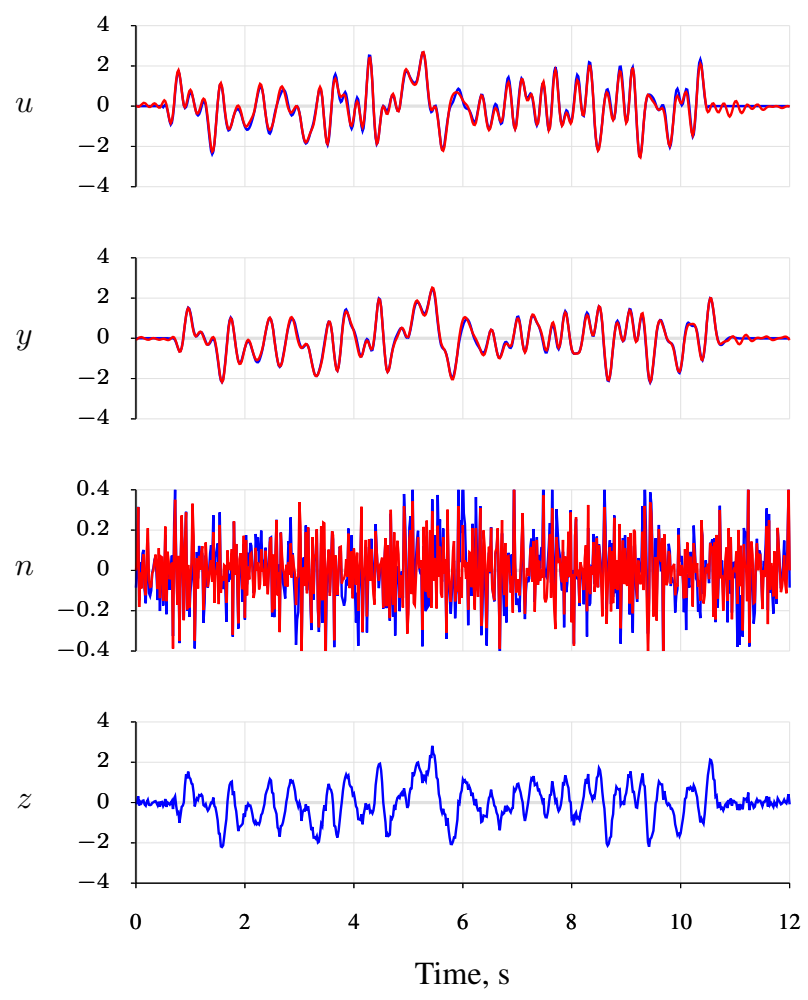

Figure 4. Simulation time series and reconstructions

above $5.5 \mathrm{~Hz}$, and smoothly attenuates the signal in between $5-5.5 \mathrm{~Hz}$. Note that the frequency spectrum in this plot and others like it in this paper are made on a logarithmic scale.

Shown also in Fig. 4 are the true and reconstructed versions of the noise, output, and input signals. The reconstructed signals were almost indistinguishable from their true signals. Coefficients of determination were $R^{2}=0.9917$ for the output signal and $R^{2}=0.9795$ for the input signal, which indicated excellent matches. The noise standard deviation was estimated within $10 \%$ of its true value. Some of the error in reconstructing the input was due to the nature of representing the constant parts of the time history near the endpoints using sinusoids. It was found using trial and error that the estimation results were not very sensitive to the general shape of the output spectral model and to the specific frequencies used to parameterize its shape in the Wiener filter, which was expected due to its robustness properties [2]. It was also found that omitting the filter and only using frequencies between $0-5.5 \mathrm{~Hz}$ in the Fourier transform, where the signal was known to have frequency content, produced good results for this case because signal-to-noise ratios were high; however, results were in general less accurate. Applying a more accurate integration method in Eq. (13), such as the trapezoidal rule, did not significantly increase the accuracy of the reconstructed input. Similarly, higher accuracy was not attained when a frequency resolution finer than $1 / T$ was used for the Fourier transform.

So far in this example, it has been assumed that the dynamic system was modeled exactly with $\hat{H}=H$. Although many systems can be modeled very accurately, there is usually some model structure error and/or parametric uncertainty in the model, which degrades the accuracy of the reconstruction. To assess the effect of parametric errors on the reconstruction, Monte Carlo runs were performed using this simulation. The noise level was varied between $0-50 \%$ of the nominal standard deviation, in $5 \%$ increments, and new noise sequences were generated for each run. The model structure of $H$ was correctly assumed, but model parameters were randomized using a uniform distribution that spanned $\pm 0-50 \%$ of the nominal values, also in $5 \%$ increments. The input contained the same power spectrum and record length, but different phase angles were chosen for each run, which resulted in different time histories [12]. Each case was run 200 times using different parameter values and different realizations of the input and noise sequences. In addition, these simulations were also performed for system natural frequencies between $1-5 \mathrm{~Hz}$ to investigate varying 


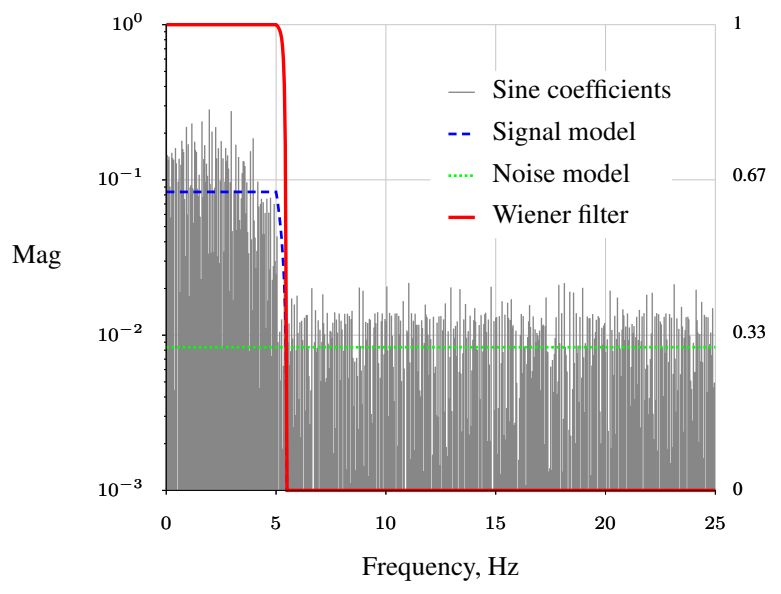

Figure 5. Spectrum of simulation data

degrees of input attenuation by the system.

Figure 6 shows the results for various levels of model uncertainty, noise level, and system natural frequency. The vertical axis in these plots indicates the $R^{2}$ value between the true input and its reconstruction. Each point on the plot surfaces represents the average of 200 simulation runs. These figures are ordered so that the system bandwidth decreases relative to the bandwidth of the input, or rather so that the higher frequencies of the input are increasingly attenuated by the system dynamics and the corresponding signal-tonoise ratios decrease. Specifically, in Fig. 6(a) the system bandwidth is equal to that of the input; in Fig. 6(b) the system bandwidth has decreased to $3 \mathrm{~Hz}$ (the nominal case); and in Fig. 6(e) the system bandwidth is $1 \mathrm{~Hz}$, so that the system attenuates most of the input. These plots indicate that, generally, if the model uncertainty is low then the input can be reconstructed accurately. If the model uncertainty increases, then the level of noise can also degrade the reconstruction. Model accuracy and high levels of signal to noise becomes more critical as the bandwidth of the input increases past the bandwidth of the system.

\section{IV.B. Integrating Noisy Data}

This example uses flight test data from the X-56A subscale aeroelastic demonstrator, pictured in Fig. 7, where the control surfaces were simultaneously excited with orthogonal phase-optimized multisines [7] over a frequency band that included the short period mode and first symmetric wing bending mode. As described in Ref. [13], the acceleration $\ddot{\eta}$ associated with the generalized bending mode coordinate was estimated using gyroscope and accelerometer data, and is shown in Fig. 8. It was desired to obtain estimates of the corresponding rate $\dot{\eta}$ and displacement $\eta$ from this data for further analysis. All numerical values were removed from the plots in this section because of ITAR restrictions with the X-56A aircraft; however, the x-axes in Figs. 8-11 span the same range of frequency, and the y-axes in Figs. 8, 9, and 11 span the same range of $\eta$.

Figure 9 shows the estimated modal position $\eta$, computed by naively integrating the acceleration twice in the frequency domain by multiplying the data with $H(j \omega)=1 /(j \omega)^{2}$. This process amplified the lowfrequency noise in the acceleration estimate, making the low-frequency portion of the displacement estimate unusable. This problem is similar to how differentiating noisy data amplifies high-frequency noise.

To improve the displacement estimate, deconvolution was applied. The signal model, noise model, and Wiener filter were constructed from Fourier sine series coefficients as shown in Fig. 10. Because the noise at low frequencies was larger than the noise at higher frequencies, a straight line was fit to these data to model the noise. A tent function was used as the signal model to approximate the bending mode response. Note that the log scaling makes the straight lines appear curved.

Figure 11 shows the estimated displacement data in the frequency domain computed using deconvolution. For comparison, displacement estimates determined using strain gauges data is also shown. The deconvolved data no longer exhibits the large low-frequency error shown in Fig. 8. Over most of the spectrum shown, the deconvolved data matches the estimates from using the strain gauge data well. However, the four lowestfrequency peaks were not accurately reconstructed by the deconvolution method. This was because the 


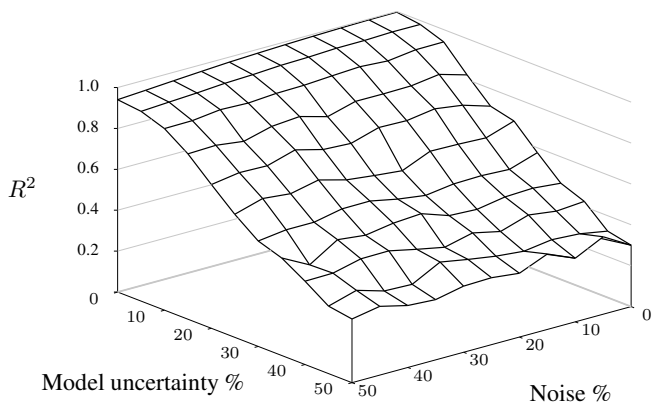

(a) $\omega_{n}=31.4 \mathrm{rad} / \mathrm{s}(5 \mathrm{~Hz})$

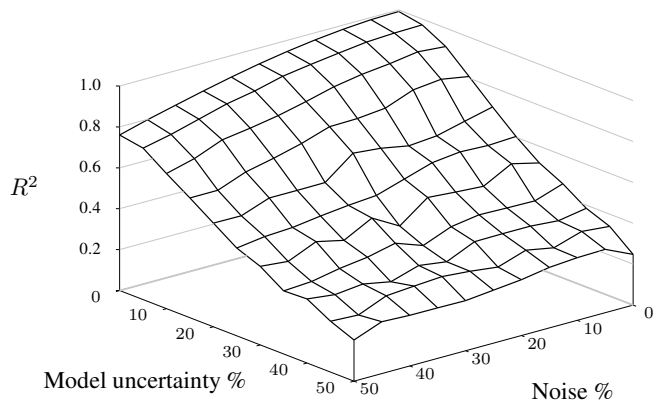

(c) $\omega_{n}=12.6 \mathrm{rad} / \mathrm{s}(2 \mathrm{~Hz})$

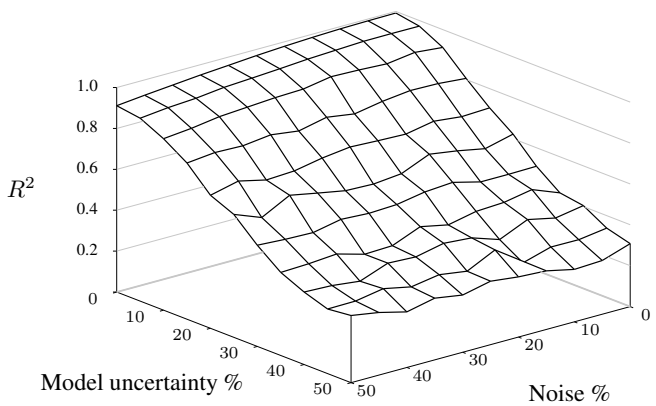

(b) $\omega_{n}=18.8 \mathrm{rad} / \mathrm{s}(3 \mathrm{~Hz})$

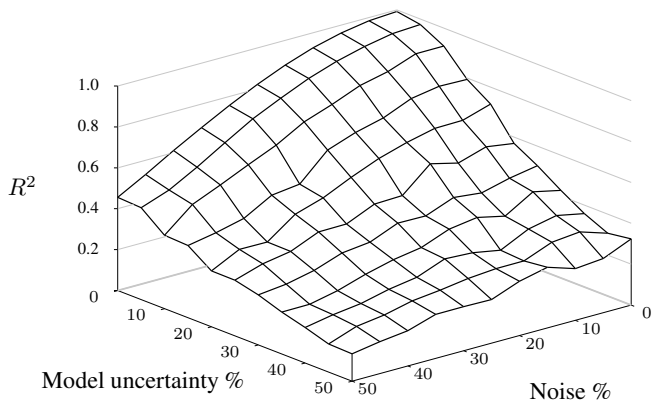

(d) $\omega_{n}=9.4 \mathrm{rad} / \mathrm{s}(1.5 \mathrm{~Hz})$

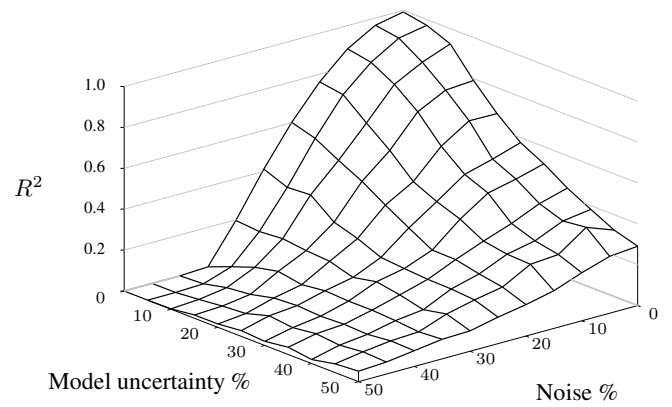

(e) $\omega_{n}=6.3 \mathrm{rad} / \mathrm{s}(1 \mathrm{~Hz})$

Figure 6. $R^{2}$ values for simulation data (200 runs averaged for each data point) 
bending mode accelerations at these frequencies were lower in magnitude than the noise model, and could not be recovered. This example illustrates a fundamental limit to using deconvolution.

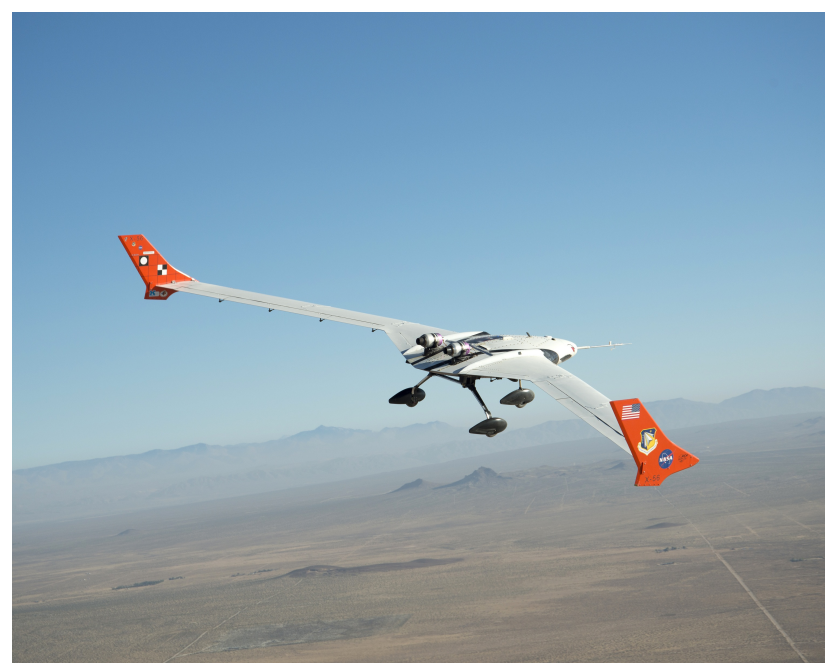

Figure 7. X-56A in flight over NASA Armstrong Flight Research Center (credit: NASA / Jim Ross)

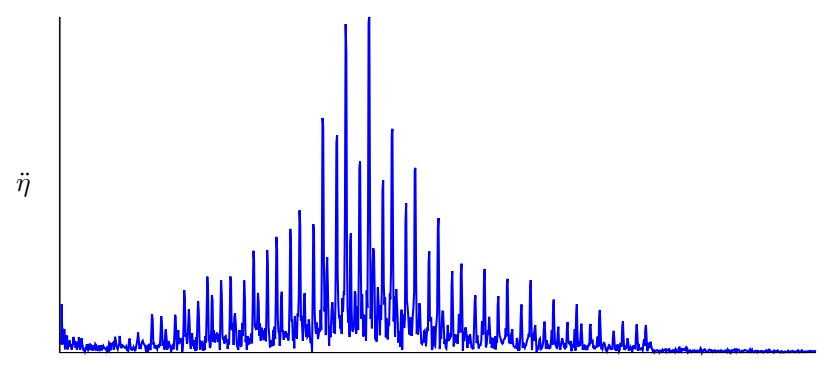

Frequency

Figure 8. Spectrum of bending mode acceleration data from X-56A flight test (Phase 1, Flight 11, FTA 503)

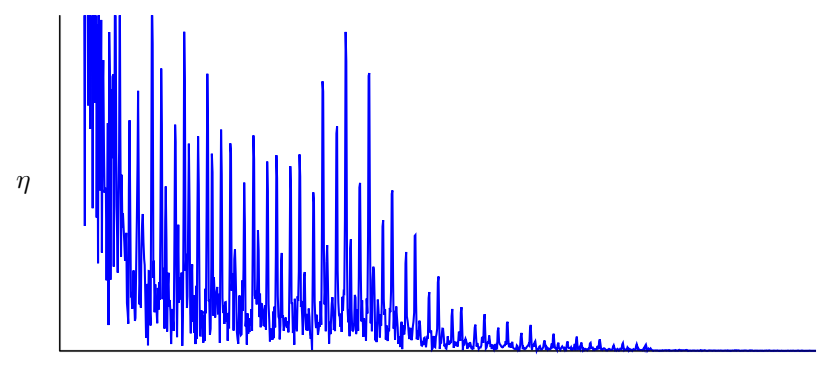

Frequency

Figure 9. Naive double integration of X-56A bending mode acceleration data 


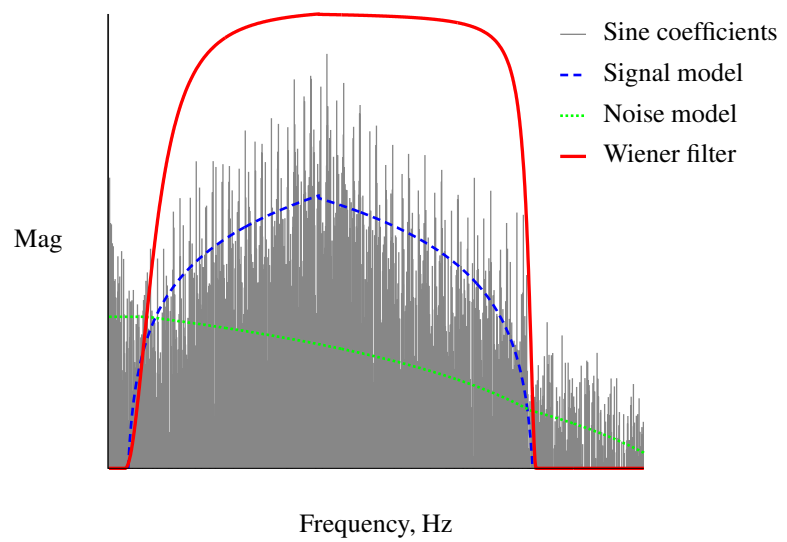

Figure 10. Spectrum of the X-56A bending mode acceleration data

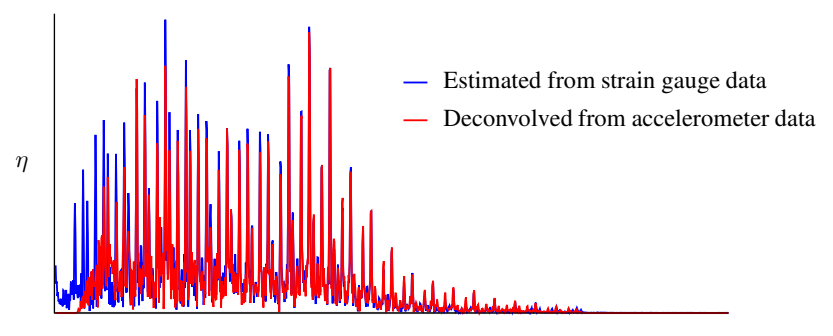

Frequency

Figure 11. Spectrum of X-56A bending mode displacement data

\section{IV.C. Synchronizing Time-Skewed Data}

Flight test data often contain relative time skews between the individual data channels that arise from various sources such as transport lags in pneumatic tubes or analog-to-digital conversion in digital systems [14]. These time skews can degrade the results of analyses, such as the estimation of stability and control derivatives [15]. For that reason, techniques have been developed to account for unknown time skews during the estimation process $[7,16-18]$. As an alternative, deconvolution can be used in some cases to synchronize data sets before estimation.

As an example, data from the NASA T-2 subscale airplane, shown in Fig. 12 with geometry and nominal mass properties listed in Table 1, was used. Onboard instrumentation included two triaxial rate gyroscopes, housed in different inertial measurement units (IMUs). It was known that the second IMU had a time delay relative to the first IMU due to internal processing and signal conditioning of the data. These delays can be seen in Fig. 13(a), where only $2 \mathrm{~s}$ of a $15 \mathrm{~s}$ maneuver are shown so that the delays are evident. The synchronized versions of these data, shown in Fig. 13(b), are discussed later in this section. In the framework of deconvolution, the input is the angular rate of the aircraft, the dynamic system is a pure time delay

$$
H(s)=e^{-s \tau}
$$

representing the low-frequency sensor dynamics of the second IMU, and the measurements are the noisy angular rate outputs. The first IMU had no significant time delay relative to the other sensor measurements. Removing the time skew and synchronizing the data sets was done by designing a Wiener filter and inverting the dynamic model in Eq. (15).

Spectral data for the pitch rate measurement are shown in Fig. 14, and were similar for the roll rate and yaw rate data. The spectrum shows the rigid-body response of the aircraft over $0-2.2 \mathrm{~Hz}$ and the noise spectrum over 0-25 Hz. The noise model was a straight line fit to the spectrum between 5-25 Hz. The signal model was a constant, fit to the average power between $0-2.5 \mathrm{~Hz}$, followed by a linear taper to zero at $3 \mathrm{~Hz}$.

To determine values of the relative time delays, output error in the frequency domain was performed using the two sets of IMU data, as described in Ref. [16]. Different time delays were estimated for each 


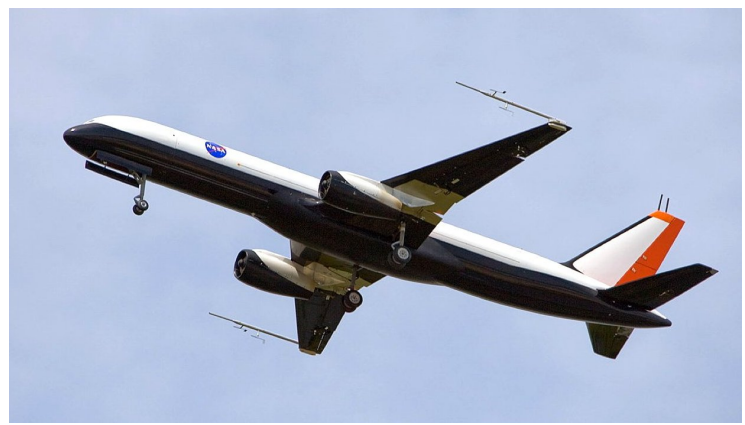

Figure 12. T-2 airplane (credit: NASA Langley Research Center)

Table 1. T-2 geometry and nominal mass properties

\begin{tabular}{ccc}
\hline \hline Parameter & Value & Unit \\
\hline$b$ & 6.85 & $\mathrm{ft}$ \\
$\bar{c}$ & 0.92 & $\mathrm{ft}$ \\
$S$ & 5.90 & $\mathrm{ft}^{2}$ \\
$x_{c m}$ & 56.4 & in \\
$z_{c m}$ & 11.5 & in \\
$m$ & 1.64 & slug \\
$I_{y y}$ & 4.65 & slug-ft \\
\hline
\end{tabular}
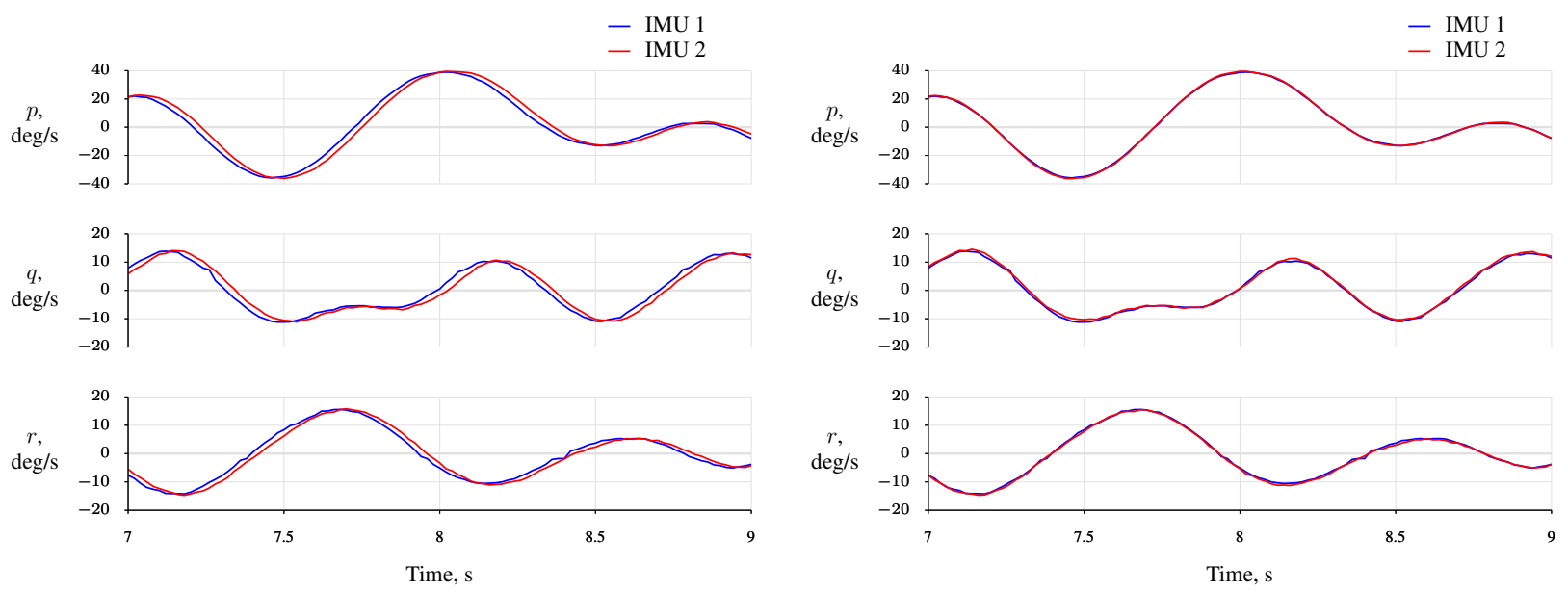

(a) Measured data with time skews

(b) Synchronized data without time skews

Figure 13. Synchronization of T-2 flight tests data (Flight 33, Maneuver 2) 


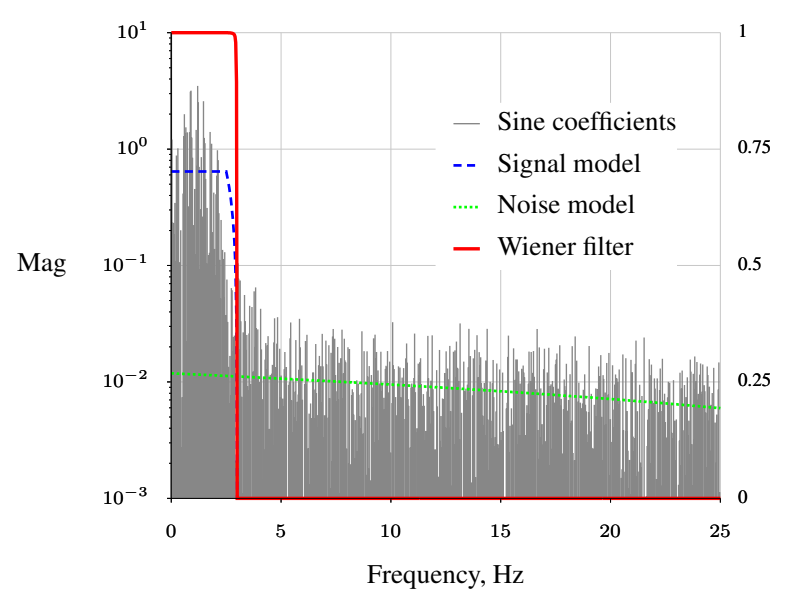

Figure 14. Spectrum of measured pitch rate data from T-2 flight test (Flight 33, Maneuver 2)

gyroscope channel, and were determined to be $0.0260 \pm 0.0005,0.0260 \pm 0.0008$, and $0.0245 \pm 0.0010 \mathrm{~s}$ for the roll, pitch, and yaw channels, respectively. These estimates had low standard errors and were close in value, as expected. Additional analysis indicated that the time delay model structure in Eq. (15) was sufficient, and that higher-order dynamics could not be accurately extracted from these flight test data or other available maneuvers.

Applying the deconvolution method synchronized the gyroscope data from the two IMUs, as shown in Fig. 13(b). The $R^{2}$ values between the two data increased from $0.98,0.96$, and 0.97 to $1.0,0.99$, and 0.99 for the roll, pitch, and yaw channels, respectively. Note that this method can shift the data by an arbitrary amount of time, which is similar in this regard to Ref. [16] but in contrast to using the sample autocorrelation function, which can only shift data by integer multiples of the sample period. The estimated noise removed by the Wiener filter can also be added back to the reconstructed signal, as was done in Fig. 13(b), for use in analyses such as output error.

\section{IV.D. Reconstructing Control Surface Deflections}

In this example, flight test data from the T-2 aircraft are used to reconstruct control surface deflections from measured output data. This example is similar to the simulation example in Section IV.A, but uses flight test data and multiple outputs. Reconstructing control surface deflections in this way could be useful in accident investigations, where control surface data are recorded at rates too low for dynamic analysis or not at all, or for when there are failures in sensors or recording systems.

To construct a dynamic model of the T-2 aircraft, five small-perturbation maneuvers flown in calm air were selected for identification. These maneuvers included simultaneous excitation of the elevator, aileron, and rudder surfaces between $0.2-2.2 \mathrm{~Hz}$ using orthogonal phase-optimized multisines; however, only longitudinal motion is studied in this example. Nondimensional stability and control derivatives for the short-period model were estimated for these combined maneuvers using equation-error parameter estimation in the frequency domain [7], and are given in Table 2. Excellent fits to model data (not shown), low standard errors on the model parameters, and agreement with previous analyses indicated that this was a good identification of the short period model. Expressed as transfer functions, the short-period model is

$$
\left[\begin{array}{c}
\Delta \alpha \\
\Delta q \\
\Delta a_{z}
\end{array}\right]=\left[\begin{array}{c}
H_{11}(s) \\
H_{21}(s) \\
H_{31}(s)
\end{array}\right] \Delta \delta_{e}
$$

where each $H_{i j}(s)$ is given in Appendix A.

Figure 15 shows flight test data for one small-perturbation maneuver not already used for model identification. Fourier sine coefficients, signal and noise models, and the Wiener filter design for the $a_{z}$ data are shown in Fig. 16. Results were similar for the $\alpha$ and $q$ data. A constant noise model was fit to the data between $5-25 \mathrm{~Hz}$, and a constant signal model was fit to the data between $0.1-2.3 \mathrm{~Hz}$, which spanned the excitation bandwidth. 
Table 2. T-2 short period modeling results using five maneuvers in calm air

\begin{tabular}{cc}
\hline \hline Parameter & Estimate \pm Std. Error \\
\hline$C_{L_{\alpha}}$ & $+4.01 \pm 0.05$ \\
$C_{L_{q}}$ & $+34.8 \pm 3.72$ \\
$C_{L_{\delta_{e}}}$ & $+0.36 \pm 0.07$ \\
$C_{m_{\alpha}}$ & $-1.47 \pm 0.03$ \\
$C_{m_{q}}$ & $-60.0 \pm 2.54$ \\
$C_{m_{\delta_{e}}}$ & $-1.81 \pm 0.05$ \\
\hline \hline
\end{tabular}
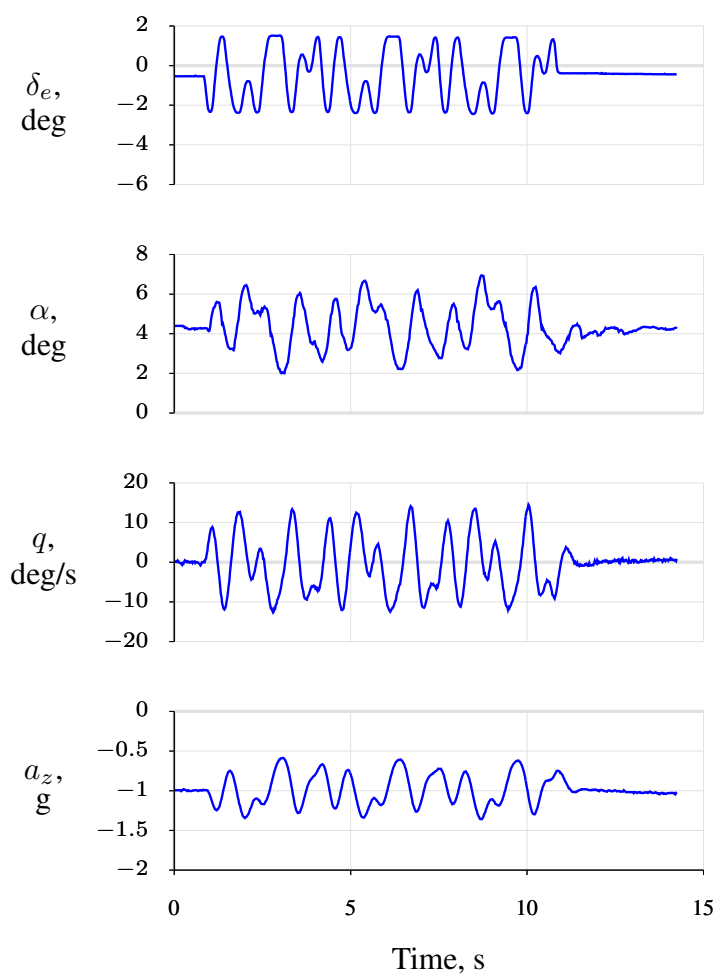

Figure 15. Measurements from T-2 flight test in calm air (Flight 33, Maneuver 4)

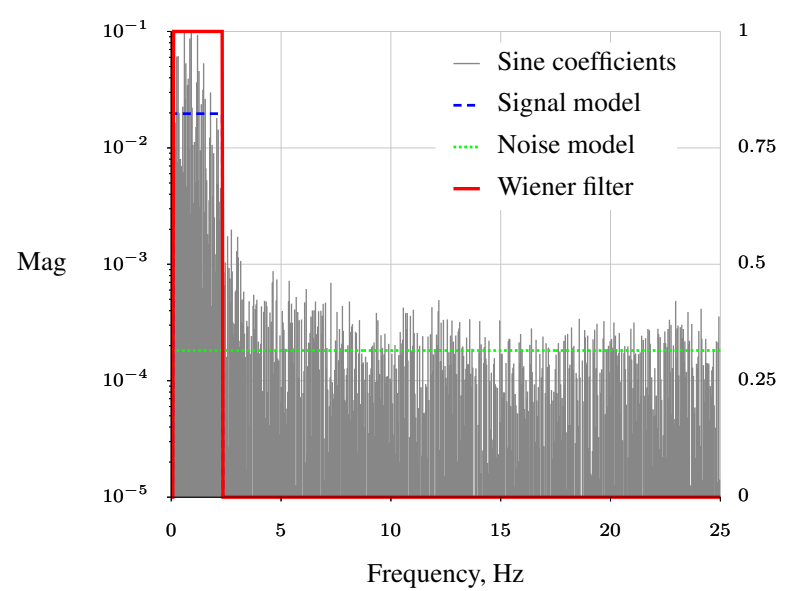

Figure 16. Spectrum of vertical accelerometer data from T-2 flight test 
In the framework of deconvolution, Eq. (16) contains three outputs and one unknown input, which makes the estimation problem over-determined. Least squares can be applied at each frequency to combine the information obtained from the three measurements and improve the estimate of the elevator deflection obtained using any single output. The least-squares solution [7] for the elevator input is

$$
\Delta \hat{\delta}_{e}=\left(\mathbf{X}^{\dagger} \mathbf{X}\right)^{-1} \mathbf{X}^{\dagger} \boldsymbol{\phi}
$$

where the regressors $\mathbf{X}$ are evaluations of the short period transfer function matrix and where $\phi$ are the measured outputs in the frequency domain.

This procedure could easily be expanded to estimate multiple inputs, such as the aileron and rudder deflections. The cost for doing this would be including additional measurements (e.g., lateral accelerometers, roll and yaw rates, and sideslip, etc.) and transfer functions; otherwise the deconvolution procedure is the same. In this case, Eq. (16) would contain additional outputs and inputs, and the transfer function matrix would be larger. There must however be at least as many outputs as inputs to obtain a solution.

Figure 17 shows the deconvolution and least-squares results. For comparison, the measured elevator time series is also shown. The fit was excellent and had $R^{2}=0.96$. Small errors are seen in matching the peaks and during portions of the maneuver where the elevator was steady.

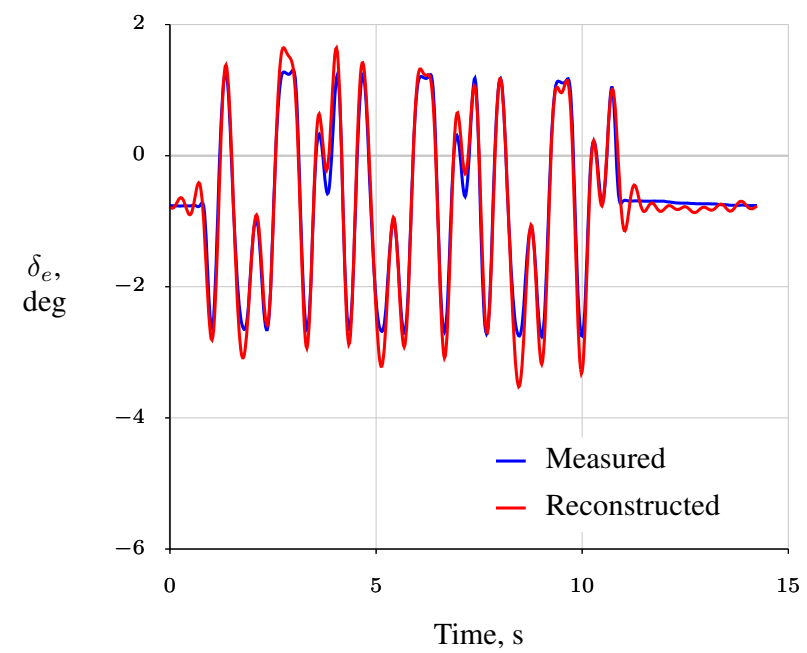

Figure 17. Measured and reconstructed T-2 elevator time series

\section{IV.E. Turbulence Estimation from Flight Test Data}

The method was also used to estimate turbulence from flight test data. Related techniques have been used for extraction of turbulence models in other works $[19,20]$. The input to be reconstructed was the turbulent gust velocity in angle of attack, the dynamic system was again the linear model of the T-2 airplane short period dynamics, and the measurements were angle of attack, pitch rate, and vertical acceleration.

The short period transfer function model was expanded as

$$
\left[\begin{array}{c}
\Delta \alpha \\
\Delta q \\
\Delta a_{z}
\end{array}\right]=\left[\begin{array}{ll}
H_{11}(s) & H_{12}(s) \\
H_{21}(s) & H_{22}(s) \\
H_{31}(s) & H_{32}(s)
\end{array}\right]\left[\begin{array}{c}
\Delta \delta_{e} \\
\Delta \alpha_{g}
\end{array}\right]
$$

to include the additional input from the angle of attack gust $\alpha_{g}$. Appendix A lists the individual transfer functions $H_{i j}(s)$. Assuming quasi-static aerodynamics, the turbulence creates an effective angle of attack [21]

$$
\alpha_{e}=\alpha+\alpha_{g}
$$

which multiplies the stability derivatives $C_{L_{\alpha}}$ and $C_{m_{\alpha}}$ in the nondimensional lift and pitching moment coefficients. Applying this model structure, dynamic models identified from flight test data in calm air (listed in Table 2) can be used to model flight in turbulent air, assuming the stability and control derivatives do not change. 
Six repeated maneuvers flown in turbulent air were selected for turbulence estimation. Data from one maneuver is shown in Fig. 18. These maneuvers were flown at approximately the same flight condition as those in calm air and discussed in Section IV.D, and were about $12 \mathrm{~s}$ in duration. The same multisine inputs were used, except the amplitudes were increased to achieve higher signal-to-turbulence ratios, and additional low frequency pilot input was applied to keep the airplane near condition. The measurements contained more high-frequency content and looked different than the corresponding responses measured in calm air, primarily in angle of attack and vertical acceleration, as seen by comparing Figs. 15 and 18.
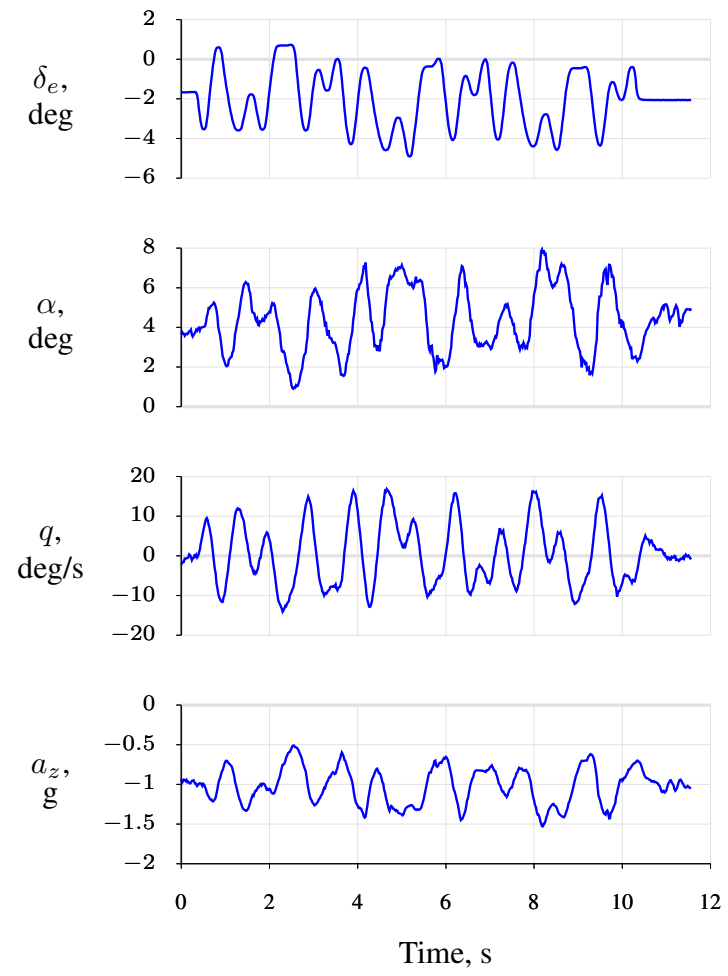

Figure 18. Measurements from T-2 flight test in turbulent air (Flight 15, Maneuver 5)

The spectral data used to design the Wiener filter are shown in Fig. 19 for the vertical accelerometer; angle of attack and pitch rate data were similar. The Fourier sine coefficients show the responses of the rigidbody dynamics at low frequencies, and then roll off with frequency to the noise floor, as is characteristic of data obtained in turbulent flight [22]. The turbulence is most evident in the vertical acceleration data, followed by the angle of attack data and pitch rate data. The noise was fitted using a straight line between 10-25 Hz. The signal was assumed to be a constant between 0-2.5 Hz, as before, and a linear taper between 2.5-10 Hz to approximate the turbulence responses. The $10 \mathrm{~Hz}$ cutoff was selected from examination of these plots on a linear scale. However, the results were not very sensitive to this value, nor to the shape of the output model (e.g., two piece-wise constant step functions were originally used for this analysis). The Wiener filters were constructed using these spectral models and applied to the respective measurements to remove the noise from the output signals.

After the noise was removed from the measured output data, least squares was again used to estimate the unknown angle of attack gust. To do this, the dynamics model was partitioned according to the two inputs and rearranged as

$$
\left[\begin{array}{c}
\Delta \alpha \\
\Delta q \\
\Delta a_{z}
\end{array}\right]-\left[\begin{array}{c}
H_{11}(s) \\
H_{21}(s) \\
H_{31}(s)
\end{array}\right] \Delta \delta_{e}=\left[\begin{array}{c}
H_{12}(s) \\
H_{22}(s) \\
H_{32}(s)
\end{array}\right] \Delta \alpha_{g}
$$

This way, the modeled response due to the measured elevator deflection was subtracted from the output data on the left side of the equation, and only the response due to the unknown gust remained on the right side. If the elevator deflection was not measured but was intended to be estimated as well, the equation 


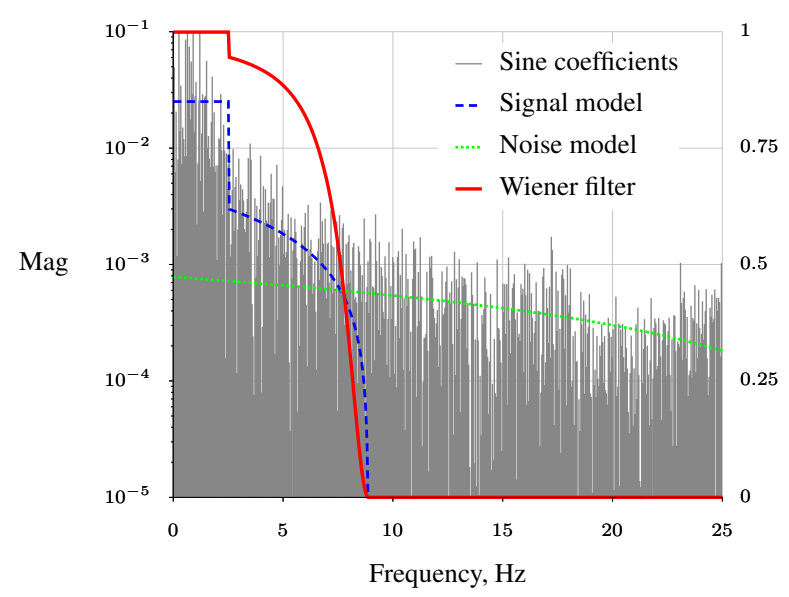

Figure 19. Spectra of measured vertical acceleration data from T-2 flight test in turbulence (Flight 15, Maneuver 5)

would not be partitioned in this way. The least-squares solution is

$$
\Delta \hat{\alpha}_{g}=\left(\mathbf{X}^{\dagger} \mathbf{X}\right)^{-1} \mathbf{X}^{\dagger} \phi
$$

where $\boldsymbol{\phi}$ is the left side of Eq. (20) and where $\mathbf{X}$ are the gust transfer functions on the right side. Other turbulence velocity inputs (e.g., side or forward gusts, or angular components) could also be estimated by using additional measurements and supplying the corresponding transfer functions to this model.

Figure 20 shows angle of attack data for one maneuver in turbulence. The red line is the measured angle of attack, and the blue line is the reconstructed angle of attack gust using deconvolution. The green line is the difference between the two time series, and represents the response of the aircraft to the elevator input only, which is similar to that shown in Fig. 15 for calm air.

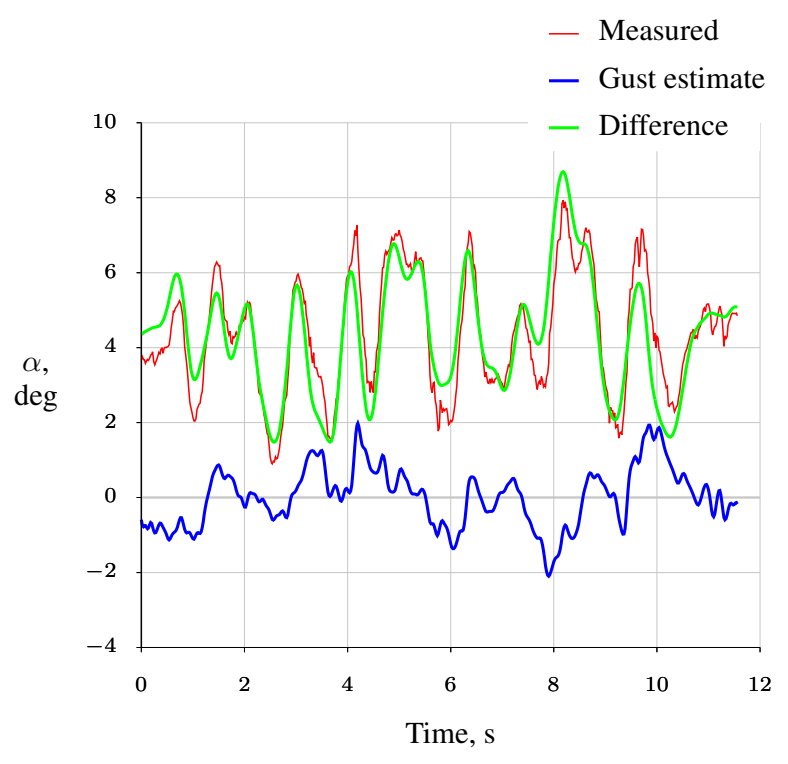

Figure 20. Angle of attack components from T-2 flight test in turbulence (Flight 15, Maneuver 5)

The power spectrum of the estimated gust velocity was also examined. The vertical gust component of the turbulence

$$
\Delta w_{g}=u_{0} \Delta \alpha_{g}
$$

was computed for easier comparison with standard turbulence models. Then the one-sided periodogram

$$
G_{w_{g} w_{g}}\left(j \omega_{k}\right)=\frac{2}{T}\left|\Delta w_{g}\left(j \omega_{k}\right)\right|^{2}
$$


was computed from $0.08-10 \mathrm{~Hz}$ and averaged at each frequency using data from the six maneuvers, consistent with the definition of the periodogram [8]. The resulting turbulence spectrum is shown in Fig. 21. A trend line was fit to this data and had slope -2.5. For reference, the von Kármán turbulence model [23] has an asymptotic slope of $-5 / 3$, or roughly -1.7 . The difference in slopes could be attributed to the type of turbulence encountered, the subscale model used for flight testing, or convective air currents experienced at low altitude in the vicinity of the testing area. A separate analysis was performed to ensure changes in the value of stability and control derivatives between flights did not significantly alter the results. A classical method for turbulence estimation [24,25], based on sensor measurements alone and not involving dynamic models, was used for comparison. Results of the classical method, using the same flight data, are also shown in Fig. 21. The results obtained using the classical method deviated from the deconvolution results near 1 $\mathrm{Hz}$. This was because the short period dynamics, occurring at approximately $1 \mathrm{~Hz}$, were not accounted for by the classical method. However, the two methods were in agreement below and above that frequency, thus corroborating the deconvolution results.

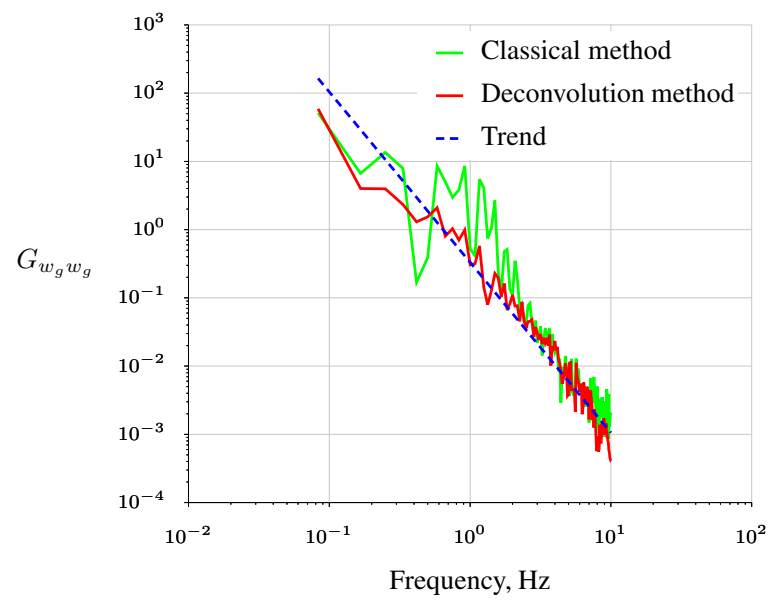

Figure 21. Estimated vertical gust power spectrum from T-2 flight test data in turbulence (Flight 15, six maneuvers)

\section{Conclusions}

This paper presented a method of frequency-domain deconvolution that uses a model of a dynamic system and noisy measurements to reconstruct the input. A Wiener filter is designed using spectral data. Techniques from Fourier analysis were adopted to increase the accuracy of this step. Then the filtered outputs are inverted by the plant dynamics model in the frequency domain, and the estimated input is transformed back into the time domain. The deconvolution method can be applied to linear, time-invariant dynamical systems that are stable or unstable, open-loop or closed-loop, single-input single-output or multiple-input multiple-output.

Deconvolution has a number of applications in the analysis of flight test data. Examples included simulation with a generic second-order system, integration of noisy data, synchronization of time-skewed data records, control surface reconstruction, and turbulence estimation. The simulation results indicated that the method performs well given potentially uncertain dynamic models and crude spectral models for the signal and noise. Analysis of Monte Carlo runs indicated that if the noise level and model uncertainty remain within about $5 \%$, the input can be reconstructed with at least $90 \%$ accuracy. Results were better for systems with higher natural frequencies where less of the input was attenuated. The first two flight test examples showed that noisy data can be integrated twice without polluting the lower frequencies, and that time-skewed data sets can be synchronized with this method. Flight test results also showed that control surface deflections or turbulence velocity inputs can be reconstructed from measured output data. 


\section{Acknowledgments}

This research was supported by the NASA Advanced Air Transport Technology project. The efforts of the X-56A team at NASA Armstrong Flight Research Center and the AirSTAR flight test team at NASA Langley Research Center are gratefully acknowledged.

\section{References}

${ }^{1}$ Morelli, E., "Estimating Noise Characteristics from Flight Test Data Using Optimal Fourier Smoothing," Journal of Aircraft, Vol. 32, No. 4, July-August 1995, pp. 689-695.

${ }^{2}$ Wiener, N., Extrapolation, Interpolation, and Smoothing of Stationary Time Series, MIT Press, 1949.

${ }^{3}$ Lánczos, C., Applied Analysis, Prentice Hall, 1956.

${ }^{4}$ Levin, A., Weiss, Y., Durand, F., and Freeman, W., "Understanding Blind Deconvolution Algorithms," IEEE Transactions on Pattern Analysis and Machine Intelligence, Vol. 33, No. 12, December 2011, pp. 2354-2367.

${ }^{5}$ Celi, R., "Optimization-Based Inverse Simulation of a Helicopter Slalom Maneuver," Journal of Guidance, Control, and Dynamics, Vol. 23, No. 2, March-April 2000, pp. 289-297.

${ }^{6}$ Morelli, E., "System IDentification Programs for AirCraft (SIDPAC)," http://software.nasa.gov, Accessed: 2016-1206.

${ }^{7}$ Morelli, E. and Klein, V., Aircraft System Identification: Theory and Practice, 2nd edition, Sunflyte Enterprises, Williamsburg, VA, 2016

${ }^{8}$ Press, W., Teukolsky, S., Vetterling, W., and Flannery, B., Numerical Recipes, Cambridge University Press, 1986.

${ }^{9}$ Bendat, J. and Piersol, A., Engineering Applications of Correlation and Spectral Analysis, John Wiley \& Sons, 2nd ed., 1993.

${ }^{10}$ Bendat, J. and Piersol, A., Random Data: Analysis and Measurement Procedures, Probability and Statistics, John Wiley \& Sons, 4th ed., 2010.

${ }^{11}$ Morelli, E., "High Accuracy Evaluation of the Finite Fourier Transform Using Sampled Data," Tech. Rep. TM-110340, NASA, Hampton, VA, June 1997.

${ }^{12}$ Grauer, J., "Random Noise Generation Using Fourier Series," Journal of Aircraft, 2018, awaiting publication, available online 22 Feb. 2018.

${ }^{13}$ Grauer, J. and Boucher, M., "Real-Time Parameter Estimation for Flexible Aircraft," No. xxxx, AIAA, Atlanta, GA, June 2018.

${ }^{14}$ Maine, R. and Iliff, K., "Application of Parameter Estimation to Aircraft Stability and Control: The Output-Error Approach," Tech. Rep. RP-1168, NASA, Edwards, CA, June 1986

${ }^{15}$ Steers, S. and Iliff, K., "Effects of Time-Shifted Data on Flight-Determined Stability and Control Derivatives," Tech. Rep. TN D-7830, NASA, Edwards, CA, March 1975.

${ }^{16}$ Morelli, E., "Dynamic Modeling from Flight Data with Unknown Time Skews," Journal of Guidance, Control, and Dynamics, Vol. 40, No. 8, August 2017, pp. 2083-2091.

${ }^{17}$ Jategaonkar, R., Flight Vehicle System Identification: A Time-Domain Methodology, Vol. 245 of Progress in Astronautics and Aeronautics, AIAA, 2nd ed., 2015.

${ }^{18}$ Tischler, M. and Remple, R., Aircraft and Rotorcraft Identification, AIAA Education Series, AIAA, 2nd ed., 2012.

${ }^{19}$ Lappi, U. and Rinaldi, L., "Final Report on the Effect of Air Turbulence on Airplane Flight Paths — Including a Spectral Density Investigation of Large-Scale Air Turbulence," Tech. Rep. TB-588-F-3, Cornell Aeronautical Laboratory, Buffalo, NY, January 1953.

${ }^{20}$ Juhasz, O., Lopez, M., Berrios, M., Berger, T., and Tischler, M., "Turbulence Modeling of a Small Quadrotor UAS Using System Identification from Flight Data," Technical Meeting on VTOL Unmanned Aircraft Systems, American Helicopter Society, Mesa, AZ, January 2017.

${ }^{21}$ Etkin, B., Dynamics of Flight, John Wiley \& Sons, 1959.

${ }^{22}$ Houbolt, J., "Atmospheric Turbulence," AIAA Journal, Vol. 11, No. 4, April 1973, pp. 421-437.

${ }^{23}$ Anon., "Flying Qualities of Piloted Airplanes," Tech. Rep. MIL-F-8785C, US Air Force, November 1980.

${ }^{24}$ Chilton, R., "Some Measurements of Atmospheric Turbulence obtained from Flow-Direction Vanes Mounted on an Airplaine," Tech. rep., NACA, Hampton, VA, November 1954.

${ }^{25}$ Notess, C. and Eakin, G., "Flight Test Investigation of Turbulence Spectra at Low Altitude Using a Direct Method for Measuring Gust Velocities," Tech. Rep. VC-839-F-1, Cornell Aeronautical Laboratory, Buffalo, NY, July 1954. 


\section{A. Transfer Functions for Short Period Approximation}

This appendix provides the transfer functions used for the short-period approximation. For simplicity, the transfer functions are written in terms of dimensional stability and control derivatives, which are related to the corresponding nondimensional parameters as

$$
\begin{aligned}
L_{\alpha}=\frac{\bar{q} S}{m V} C_{L_{\alpha}} & L_{q}=\frac{\bar{q} S}{m V} \frac{\bar{c}}{2 V} C_{L_{q}} & L_{\delta_{e}}=\frac{\bar{q} S}{m V} C_{L_{\delta_{e}}} \\
M_{\alpha}=\frac{\bar{q} S \bar{c}}{I_{y y}} C_{m_{\alpha}} & M_{q}=\frac{\bar{q} S \bar{c}}{I_{y y}} \frac{\bar{c}}{2 V} C_{m_{q}} & M_{\delta_{e}}=\frac{\bar{q} S \bar{c}}{I_{y y}} C_{m_{\delta_{e}}}
\end{aligned}
$$

All values on the right side of these equations correspond to the reference condition.

Each element in the transfer function matrix is of the form

$$
H_{i j}(s)=\frac{N_{i j}(s)}{\Delta(s)}
$$

where the denominator is

$$
\Delta(s)=s^{2}+\left(L_{\alpha}-M_{q}\right) s-L_{\alpha} M_{q}-\left(1-L_{q}\right) M_{\alpha}
$$

and the numerators are

$$
\begin{aligned}
& N_{11}(s)=-L_{\delta_{e}} s+L_{\delta_{e}} M_{q}+\left(1-L_{q}\right) M_{\delta_{e}} \\
& N_{12}(s)=s^{2}-M_{q} s \\
& N_{21}(s)=M_{\delta_{e}} s+L_{\alpha} M_{\delta_{e}}-L_{\delta_{e}} M_{\alpha} \\
& N_{22}(s)=M_{\alpha} s \\
& N_{31}(s)=-\frac{V}{g}\left[L_{\delta_{e}} s^{2}+\left(L_{q} M_{\delta_{e}}-L_{\delta_{e}} M_{q}\right) s+\left(L_{\alpha} M_{\delta_{e}}-L_{\delta_{e}} M_{\alpha}\right)\right] \\
& N_{32}(s)=-\frac{V}{g}\left[L_{\alpha} s^{2}+\left(L_{q} M_{\alpha}-L_{\alpha} M_{q}\right) s\right]
\end{aligned}
$$

